\title{
Molecular characterization of inversion breakpoints in the Drosophila nasuta species group
}

\author{
Dat Mai \& Doris Bachtrog
}

Department of Integrative Biology, University of California Berkeley, Berkeley, CA 94720, USA

Chromosomal inversions are fundamental drivers of genome evolution. In the Drosophila genus, inversions have been widely characterized cytologically, and play an important role in local adaptation. Here, we characterize chromosomal inversions in the Drosophila nasuta species group using chromosome-level, reference-quality assemblies of seven species and subspecies in this clade. Reconstruction of ancestral karyotypes allowed us to infer the order in which the 22 identified inversions occurred along the phylogeny. We found a higher rate of inversions on the $\mathrm{X}$ chromosome, and heterogeneity in the rate of accumulation across the phylogeny. We molecularly characterize the breakpoints of six autosomal inversions, and found that repeated sequences are associated with inversion breakpoints in four of these inversions, suggesting that ectopic recombination is an important mechanism in generating inversion. Characterization of inversions in this species group provides a foundation for future population genetic and functional studies in this recently diverged species group.

\section{Introduction}

Inversion polymorphisms have been studied extensively in Drosophila genetics since their first discovery over a century ago (Sturtevant 1917). Chromosomal inversions were first identified as suppressors of recombination in Drosophila melanogaster (Sturtevant 1917), and characterized subsequently in detail as structural alterations in polytene chromosomes across the Drosophila genus (Krimbas and Powell 1992).

Over the past century, chromosomal inversions have been recognized as a ubiquitous evolutionary phenomenon. Inversions are present in virtually all species and can have wideranging evolutionary effects. Inversions can help maintain coadapted gene complexes, reduce gene flow in hybrid zones, or restrict recombination between diverging sex chromosomes (Hoffmann and Rieseberg 2008). In addition to modifying the recombination landscape along a chromosome, inversions can also directly alter the structure or expression of genes found near inversion breakpoints (Calvete et al. 2012; Guillén and Ruiz 2012).

Despite being ubiquitous in nature and their putative widespread consequences, the evolutionary forces maintaining inversions are typically poorly understood. Several lines of evidence suggest that many inversions found in Drosophila and other species are adaptive. In particular, inversions often show seasonal, altitudinal and/or latitudinal clines, and polymorphic inversions are often associated with fitness-related traits (Hoffmann and Rieseberg 2008; Hoffmann, Sgrò, and Weeks 2004). 
Genome-wide alignments between species allow us not only to detect the presence of chromosomal inversions but also to identify and characterize inversion breakpoint regions (Feuk et al. 2005; Ranz et al. 2007). Breakpoint sequences may shed light on the causes generating the inversion as well as on the functional consequences that the inversion might have had.

Here we identify chromosomal inversions and characterize their breakpoints in the $D$. nasuta subgroup. This species group contains about a dozen species that are distributed across SouthEast Asia. The karyotype of $D$. nasuta species consists of the X (Muller A), a large metacentric autosome (chromosome 2; Muller B, E) and a large acrocentric autosome (chromosome 3; Muller C, D), and the small dot chromosome. In D. albomicans, chromosome 3 fused to both the $\mathrm{X}$ and the $\mathrm{Y}$ chromosome, forming a neo-sex chromosome. Inversion polymorphism of the nasuta species group has been studied using cytogenetic techniques (Casu 1990; Lambert 1982;Pope 1987), and species in this group were found to be highly polymorphic for chromosomal inversions. However, no systematic characterization of inversions at the molecular level exists. Here we take advantage of high-quality chromosome-level genome assemblies for molecular characterization of inversions in the D. nasuta subgroup.

\section{Results}

\section{Karyotype evolution in the $D$. nasuta subgroup.}

Ancestral linkage groups are conserved across the Drosophila genus and termed Muller elements (Muller 1940). Most flies in the $D$. nasuta subgroup have a conserved karyotype with a telocentric $X$ (Muller A), a large metacentric autosome (Muller B and Muller E) and a telocentric autosome (Muller C and Muller D), and the small dot chromosome (Muller F). In $D$. albomicans, the telocentric autosome fused to both the ancestral $X$ and $Y$, forming a neo- $X$ and neo-Y chromosome. Our high-quality assemblies recovered each chromosome arm as a single contig (Mai, Nalley, and Bachtrog 2020). Figure 1 gives an overview of global syntenic relationships across the species investigated, based on the location of protein-coding genes; genes are assigned to Muller elements (and color-coded accordingly). Consistent with previous

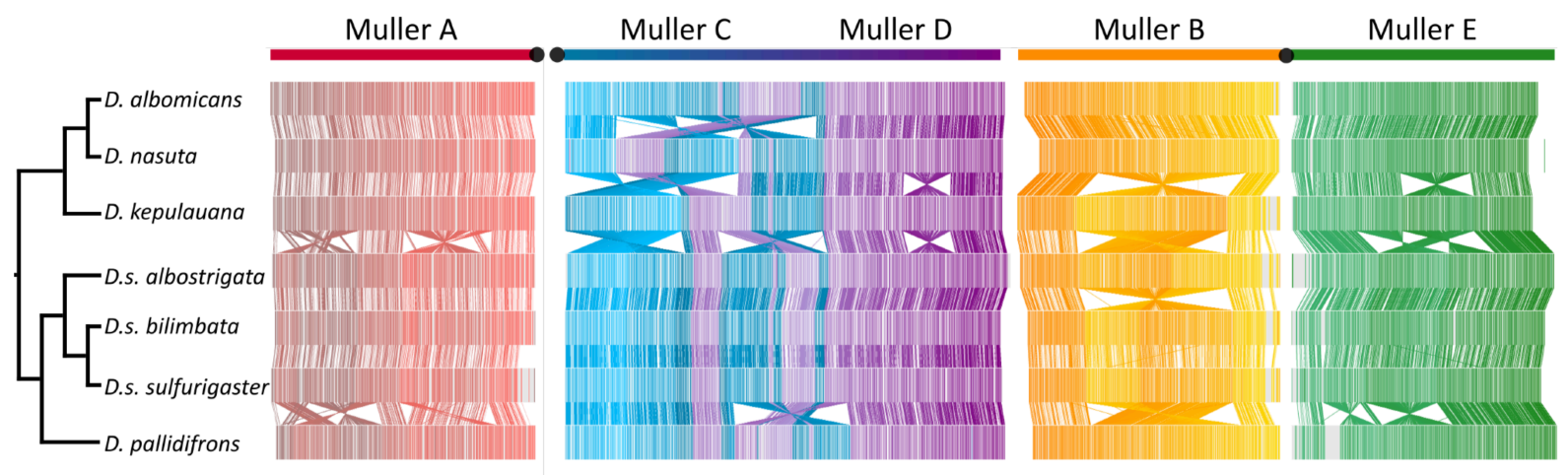

Figure 1. - Phylogenetic relationship between species investigated and chromosomal synteny based on alignments of orthologous single-copy genes. Genes are color-coded according to their assignments to Muller elements. Note that Muller A and C/D are fused in D. albomicans. 
studies within the Drosophila genus, syntenic comparisons on all Muller elements reveal a rich history of intrachromosomal reshuffling of genes (Bhutkar et al. 2008; Figure 1). Interestingly, while Muller $C$ and Muller $D$ genes are mixed up along the telocentric chromosome 3, no shuffling of Muller B and Muller E genes occurred on the metacentric chromosome 2 . This suggests that paracentric inversions are more frequent in this group than pericentric inversions, consistent with observations in other Drosophila groups (Sperlich and Pfriem 1986; Krimbas and Powell 1992).
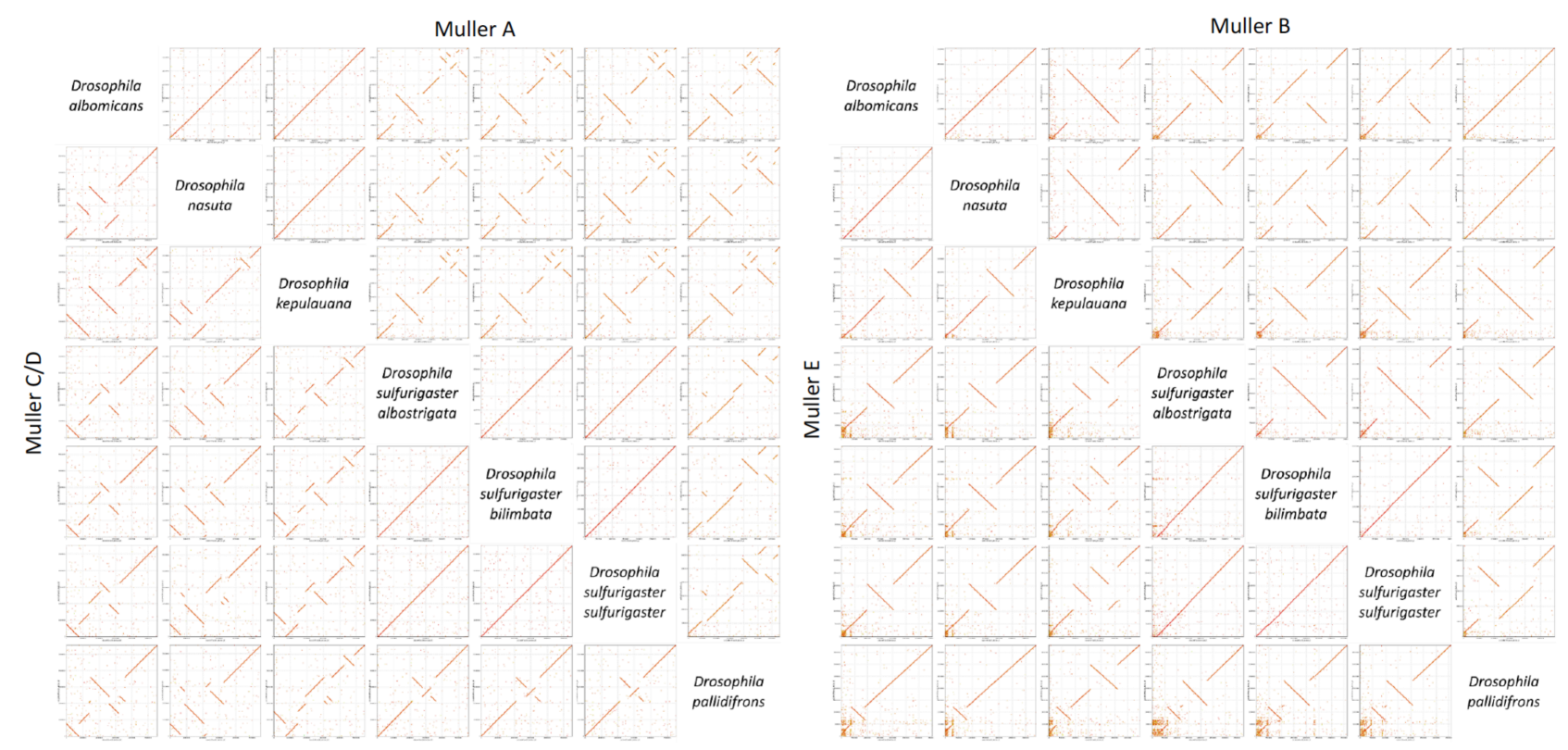

Figure 2. - Dotplot between species for all major chromosome arms (Muller elements A through $\mathrm{E}$ ). The pericentromere of each chromosome arm is placed at the bottom left corner of each subplot. Note that chromosomes are not drawn to scale (i.e. Muller C/D is approximately twice as large as all other chromosome arms).

\section{Identification of inversions using whole-genome alignments.}

We used whole-chromosome alignments to identify inversions on each chromosome arm for species of the $D$. nasuta subgroup. We used MUMmer to compare the chromosomes of each species and used breaks in synteny to map inversion breakpoints (Kurtz et al. 2004; Figure 2). For each chromosome arm, we identified syntenic segments and we used GRIMM to find the minimum number of rearrangements required to account for the order and orientation of syntenic segments along the phylogeny (Tesler 2002). In total, we identify 22 large chromosomal inversions along the major chromosomes (Figure 3). We identify 8 inversions on the ancestral $X$ chromosome (Muller A), 6 inversions on the metacentric chromosome 2 ( 3 on Muller $B$ and 3 on Muller $E$ ), and 8 inversion on the telocentric chromosome 3 (Muller $C$ and Muller D). Therefore, while encompassing only a single Muller element and thus being substantially smaller than other chromosomes, the $X$ has a similar number of inversions. Higher rates of X-linked inversions have also been found in primates (Porubsky et al. 2020). The chromosomal inversions identified vary dramatically in size, ranging from 3.9-18.0 Mb, and contain hundreds or thousands of genes (Table 1). 


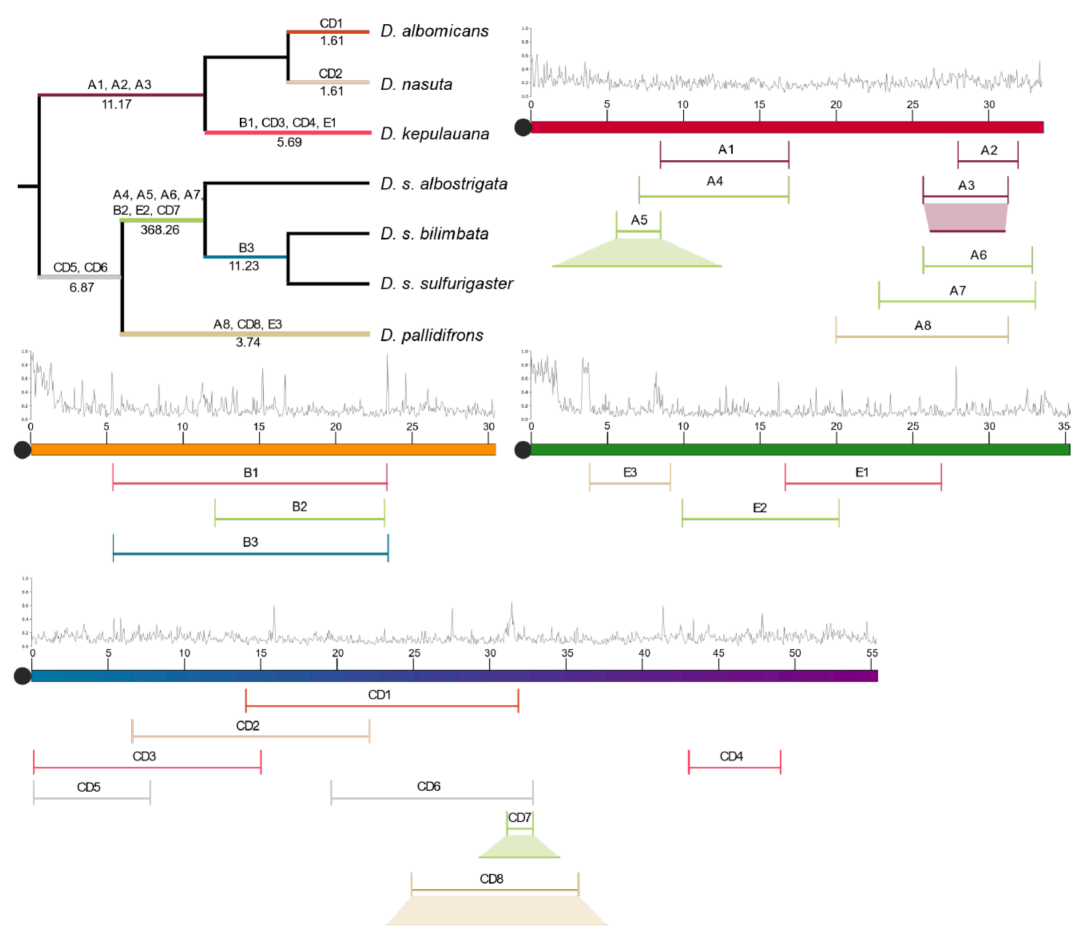

Figure 3. - Major inversions along the phylogeny based on parsimony reconstruction. The approximate location of inversions along the ancestral chromosomes is indicated, and their size (the size of nested inversions is indicated under the shaded region). Inversions are color coded by the branch on the phylogeny where they occurred. Dots indicate location of the centromere and ticks mark every $5 \mathrm{Mb}$. The repeat content (fraction repeat masked in 50-kb windows) is shown above each chromosome. The number of inversions per million years is shown along each branch (bottom) of the phylogeny.

\section{Phylogenetic reconstruction of inversion}

We reconstructed the evolution of inversions in the nasuta clade along the phylogeny using parsimony. Figure 3 shows the inferred occurrence of inversions along different branches. Our sequenced strains of $D$. albomicans and $D$. nasuta differ by two overlapping inversions on Muller C/D (which forms the neo-sex chromosome in D. albomicans), but are otherwise colinear. Their sister species $D$. kepulauana harbors two additional inversions on Muller $C / D$, and one on Muller B and Muller $E$, and this entire clade shares three inversions on Muller $A$. The sister species $D$. s. sulfurigaster and D. s. bilimbata are entirely collinear, and a single shared inversion on Muller B distinguishes them from their sister clade D. s. albostrigata. The sulfurigaster clade has four inversions on Muller $A$ in common, and on each on Muller $B, E$ and $C / D$. Their sister species D. pallidifrons has one inversion on Muller $A, C D$ and $E$, and two inversions on Muller C/D occurred in the common ancestor of the sulfurigaster flies and $D$. pallidifrons.

Overall, we find the average inversion rate to be 5.2 inversions per million years, consistent with previously found inversion rates in Drosophila (Ranz et al. 2007; Lemeunier and Ashburner 1984; Powell 1997; Vieira et al. 1997; Bartolomé and Charlesworth 2006; Papaceit, Aguadé, and Segarra 2006; González, Casals, and Ruiz 2007; Bhutkar et al. 2008). However, there is high variation in the inversion rate per branch on the phylogeny (Figure 3). In particular, almost $1 / 3$ of all inversions were identified on the short branch leading to species of the sulfurigaster species group. 
In addition, inversions appear to be more common on the $\mathrm{X}$ chromosome compared to autosomes. While encompassing only about $1 / 5$ of the total genome size, the $\mathrm{X}$ chromosome harbors more than $1 / 3$ of all the inversions detected (Figure 3 ). Again, higher rates of inversions on the $\mathrm{X}$ chromosome are consistent with previous observations in Drosophila (Cheng and Kirkpatrick 2019).

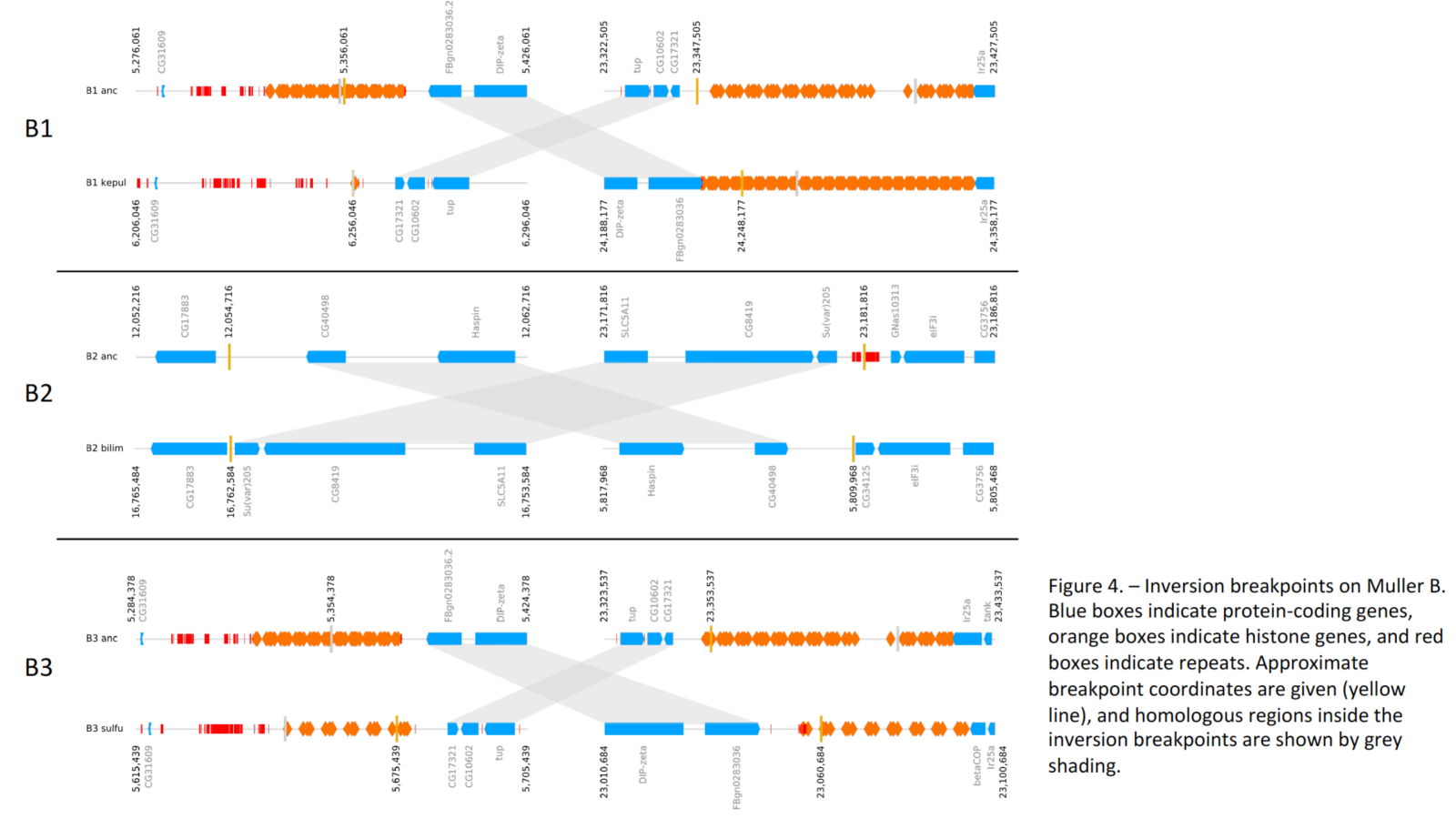

\section{Molecular characterization of breakpoints}

Localizing the precise inversion breakpoints can be informative for several reasons. Inversions may directly impact gene structure or gene expression, and the identification of inversion breakpoints might provide insights into the molecular mechanisms by which inversions arise. We therefore carefully characterized all the inversion breakpoints on Muller B and Muller E.

We identify three inversions on Muller B (Figure 4). Inversion B1 (which occurred along the $D$. kepulauana branch) and B3 (occurring along the D. s. bilimbata/D. s. sulfurigaster branch) are about $18 \mathrm{Mb}$ in size. B1 and B3 occurred at homologous positions in the genome, and both of their breakpoints are located within the histone gene cluster. Nonalleleic homologous recombination could promote recurrent generation of inversions at the histone cluster, but it is also possible that this inversion was inherited from a common ancestor. Inversion B2 is about $11 \mathrm{Mb}$ long and shared by all sulfurigaster flies. One breakpoint of this inversion is located next to HP1 (Su(var)205), an important structural component of heterochromatin, but no repeated sequences are found at the breakpoints of the inverted chromosome (Figure 4).

Muller E harbors three inversions (Figure 5). Inversion E1 is about $10 \mathrm{Mb}$ in size and occurred along the lineage leading to $D$. kepulauana. One of the breakpoints occurred at an approximately $1.6 \mathrm{~kb}$ repeat-masked region with no known homology aside from a $64 \mathrm{bp}$ 
stretch that is homologous to R1-3_DF-a non-LTR retrotransposon. The other breakpoint is in a unique region (Figure 5).

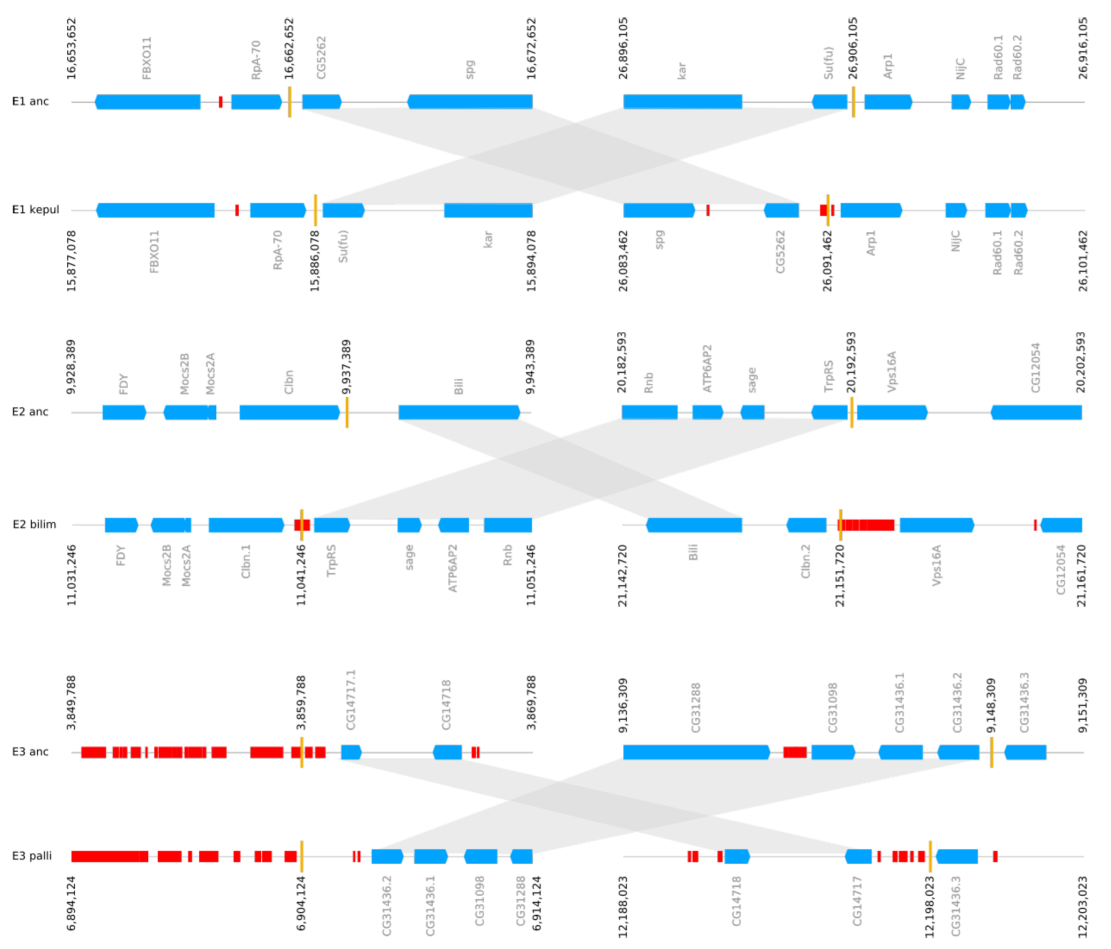

Figure 5. - Inversion breakpoints on Muller E. For legend, see Fig. 4

Inversion E2 is about $10 \mathrm{Mb}$ in size and occurred in the sulfurigaster lineage shared by $D$. $s$. albostrigata, D. s. bilibmata, and D. s. sulfurigaster. Both inversion breakpoints lie inside repetitive regions that are $1.2 \mathrm{~kb}$ and $13 \mathrm{~kb}$ in size (Figure 5). The breakpoint has a duplication of the Clbn gene along the sulfurigaster lineage while all other species in the nasuta clade have only a single copy of $\mathrm{Clbn}$, suggesting that the inversion created a duplicate copy of this gene. Duplications of non-repetitive DNA at inversion breakpoints can be caused by staggered singlestrand DNA breaks and repair by non-homologous end-joining (Guillén and Ruiz 2012). Inversion E3 occurred on the lineage leading to $D$. pallidifrons and is about $5 \mathrm{Mb}$ in size. One inversion breakpoint is found inside a large (over $1 \mathrm{Mb}$ long) repeat island, which in the $D$. pallidifrons genome is comprised of a number of transposons, over half of which are hAT elements. The other breakpoint is located within the tandemly duplicated multicopy gene CG31436. Thus, repeated sequences are found recurrently at inversion breakpoints in the $D$. nasuta species group.

\section{Discussion}

Inversion polymorphism has been studied for over a century in Drosophila. Inversions can have profound biological influences (see introduction), but the evolutionary processes maintaining inversions are typically poorly understood. Individuals heterozygote for inversions may suffer reduced fertility by producing nonfunctional gametes during meiosis. These fertility effects are expected to be less pronounced in Drosophila, since males generally lack meiosis, and aberrant 
recombinant products contribute preferentially to the polar body nurse cells in females (Reis et al. 2018).

Large differences in rearrangement rates have been reported between species and between chromosomes in Drosophila. We find dramatic variation in the rate of chromosomal inversions among lineages. Seven out of the 22 inversions identified map to the short branch that leads to flies of the sulfurigaster species complex, but only a single inversion on the branch leading to $D$. albomicans or D. nasuta (Figure 3). This is in agreement with previous observations in Drosophila, which found that rates of chromosomal inversions can differ by over an order of magnitude even among closely related species and between Muller's elements (Ranz et al. 2007; Lemeunier and Ashburner 1984; Powell 1997; Vieira et al. 1997; Bartolomé and Charlesworth 2006; Papaceit, Aguadé, and Segarra 2006; González, Casals, and Ruiz 2007; Bhutkar et al. 2008). This asymmetry in rates of inversions could result from differences in fitness effects or the efficacy of selection to establish new inversions, or from differences in mutation rates among lineages.

The molecular mechanisms of how inversions are generated are incompletely understood, and may differ among species or chromosomes. Inversions can be generated by nonallelic homologous recombination between repeated sequences, or by chromosome breakage and erroneous repair of the break by nonhomologous end-joining (Sonoda et al. 2006). Most inversions breakpoints in the melanogaster subgroup are associated with inverted duplication of genes or other non-repetitive sequences (Ranz et al. 2007). The presence of inverted duplications associated with inversion breakpoint regions was suggested to result from staggered breaks, followed by non-homologous end-joining. On the other hand, several studies in the Drosophila subgroup have found that repetitive elements are associated with the formation of inversion, suggesting an important role of ectopic exchange (Cacares et al. 1999; Fonseca et al. 2012). In the D. nasuta subgroup, we find evidence for both processes.

Reuse of inversion breakpoints in Drosophila has been reported at both the cytological and molecular level (Dobzhansky and Socolov 1939; Krivshenko 1963; Coluzzi et al. 1979; Lemeunier and Ashburner 1984; Pevzner and Tesler 2003; Zhao et al. 2004; Murphy et al. 2005; Richards et al. 2005; Goidts et al. 2005). We find that the histone gene cluster, which is located on two separate regions on Muller $B$ in flies of the nasuta subgroup was involved in the generation of inversions in two separate lineages (though we cannot rule out that this inversion was segregating in a common ancestor of this species group). This resembles findings in great apes, where a high rate of homoplasy of inversions was observed (Porubsky et al. 2020). Reuse of inversion breakpoints might be due to mutational bias if these regions are particularly prone to breakage, or driven by selection if a specific breakpoint position affects the intrinsic fitness of a new arrangement (McBroome et al. 2020). Mutations caused by inversion breakpoints may have diverse consequences, from gene disruptions to generation of new gene duplicates or transfer of regulatory sequences from one gene to another. We identify one instance of a gene duplication generated by an inversion on Muller $\mathrm{E}$ in the sulfurigaster clade. 
Chromosomal inversions can maintain linkage among alleles that are favored by natural selection and inversions that are associated with complex polygenic phenotypes are known from a variety of taxa (Hoffmann and Rieseberg 2008). Species from the nasuta clade are recently diverged, but differ in various morphological and behavioral phenotypes (Kitagawa et al. 1982; Spieth 1969). It will be of great interest to address the role of chromosomal inversions in contributing to phenotypic differences and local adaptation.

\section{Methods}

\section{Genome Assemblies \& Annotations}

We used chromosome-level assemblies for seven species of the $D$. nasuta species group, which are described elsewhere (Wei, Mai et al, in preparation). Table S1 lists the strains that were investigated. All assemblies are highly contiguous ( $\mathrm{N} 50$ s ranging from $34 \mathrm{Mb}$ to $38 \mathrm{Mb}$ ) and very complete (with BUSCO scores ranging from $98.5 \%$ to $99.7 \%$ ), and total assembly sizes ranging from $161 \mathrm{Mb}$ to $163 \mathrm{Mb}$. For each species, the euchromatic portion of all Muller elements are assembled as a single contig, and only highly repetitive pericentromeric fragments could not be placed on the assembly (the assemblies comprise between 77 to 282 scaffolds with a mean of 157 scaffolds).

Gene annotations for each species (from Wei, Mai et al, in preparation) were clustered with $D$. virilis gene annotations using OrthoDB (Kriventseva et al. 2019). We then assign a name to each gene based on clustering with $D$. virilis annotations and their homology to $D$. melanogaster genes. An average of 10,534 genes were assigned to a $D$. melanogaster gene; 10,336 of these are single copy genes and 198 are duplicated genes.

\section{Inversion Along Phylogeny}

MUMmer was used to determine the inversion status between all genome assemblies using the D. albomicans assembly as the reference (Kurtz et al. 2004). Sequences between each inversion breakpoint are assigned a numeric identifier and an optional negative sign to denote an inverted status relative to $D$. albomicans for each genome, which are then represented by an ordered sequence of these identifiers. The numeric sequence for each genome is then used as input for GRIMM to determine the optimal rearrangement scenario along a phylogeny, and identify the most likely ancestral genome structure (Tesler 2002).

We generated an "ancestral genome" by using the $D$. albomicans genome assembly, and "uninverting' all the inversions occurring along the branches leading to $D$. albomicans (that is, inversion $A 1, A 2, A 3, C D 1$; see Figure 3). To call inversion breakpoints, we took the mean between the end of the alignment on one side of the inversion and the start of the alignment on the other side of the alignment from MUMmer coordinates (Table S2).

All genome assemblies were then aligned to the ancestral genome using MUMmer. For each chromosome, all different inversion breakpoints are used to demarcate regions along the chromosome and inversions along the genome were then estimated using GRIMM based on the order and orientation of these regions relative to the ancestral genome. 


\section{Inversion Rates}

To test the rates of inversions along the phylogeny, we also calculate the branch lengths along the phylogeny. They are determined using the same method as Mai et al. 2019, using mean Ks values between species, a neutral mutation rate estimate of $3.46 \times 10^{-9}$ per base per generation, and a 7 generation per year estimate for Drosophila (Zhang et al. 2006; Cutter 2008; Keightley et al. 2009). Internal branch lengths are calculated by subtracting the divergence time between sister species from the divergence time between the mean of the sister species and a outgroup. For example, let $d_{A B}$ be the divergence time between species $A$ and B. Given the phylogeny ( $A$, $B), C)$, the length of the branch from the root node to the shared node between $A$ and $B$ is calculated by $\frac{d_{A C}+d_{B C}}{2}-d_{A B}$.

The overall inversion rate is calculated using the total number of inversions on the phylogeny divided by the total length, in millions of years, of the phylogeny (in other words, the sum of all branches along the phylogeny). The inversion rate per branch is calculated by dividing the number of inversions occurring on the branch by the branch length.

\section{Acknowledgements}

We acknowledge Ryan Bracewell for discussion.

\section{References}

Bartolomé, Carolina, and Brian Charlesworth. 2006. "Rates and Patterns of Chromosomal Evolution in Drosophila Pseudoobscura and D. Miranda." Genetics 173 (2): 779-91. https://doi.org/10.1534/genetics.105.054585.

Bhutkar, Arjun, Stephen W. Schaeffer, Susan M. Russo, Mu Xu, Temple F. Smith, and William M. Gelbart. 2008. "Chromosomal Rearrangement Inferred From Comparisons of 12 Drosophila Genomes." Genetics 179 (3): 1657-80. https://doi.org/10.1534/genetics.107.086108.

Caceres M, Ranz JM, Barbadilla A, Long M, Ruiz A. 1999. Generation of a widespread Drosophila inversion by a transposable element. Science 285(5426):415-418.

Calvete, Oriol, Josefa González, Esther Betrán, and Alfredo Ruiz. 2012. "Segmental Duplication, Microinversion, and Gene Loss Associated with a Complex Inversion Breakpoint Region in Drosophila." Molecular Biology and Evolution 29 (7): 1875-89. https://doi.org/10.1093/molbev/mss067.

Campbell, M.S., C. Holt, B. Moore, and M. Yandell. 2014. “Genome Annotation and Curation Using MAKER and MAKER-P." Curr Protoc Bioinformatics 48 (4).

Casu, R. E. 1990. "Inversion Polymorphism in Populations of Drosophila Sulphurigaster Albostrigata and Drosophila Nasuta Albomicans from Phuket, Thailand." Genetica 81 (3): 157-69. https://doi.org/10.1007/BF00360861.

Chakraborty, Mahul, James G. Baldwin-Brown, Anthony D. Long, and J. J. Emerson. 2016. "Contiguous and Accurate de Novo Assembly of Metazoan Genomes with Modest Long Read Coverage." Nucleic Acids Research 44 (19): e147. https://doi.org/10.1093/nar/gkw654.

Cheng C, Kirkpatrick M. Inversions are bigger on the X chromosome. Mol Ecol. 2019 Mar;28(6):1238-1245. doi: 10.1111/mec.14819. Epub 2018 Aug 29. PMID: 30059177; PMCID: PMC6353710.

Coluzzi, M., A. Sabatini, V. Petrarca, and M. A. Di Deco. 1979. "Chromosomal Differentiation and Adaptation to Human Environments in the Anopheles Gambiae Complex." Transactions of the Royal Society of Tropical Medicine and Hygiene 73 (5): 483-97. https://doi.org/10.1016/0035-9203(79)90036-1.

Cutter, A.D. 2008. "Divergence Times in Caenorhabditis and Drosophila Inferred from Direct Estimates of the Neutral Mutation Rate." Mol. Biol. Evol 25: 778-786.

Dobzhansky, Th., and D. Socolov. 1939. "STRUCTURE AND VARIATION OF THE CHROMOSOMES IN DROSOPHILA AZTECA." Journal of Heredity 30 (1): 3-19. https://doi.org/10.1093/oxfordjournals.jhered.a104629. 
Dudchenko, O., S.S. Batra, A.D. Omer, S.K. Nyquist, M. Hoeger, N.C. Durand, M.S. Shamim, I. Machol, E.S. Lander, and A.P. Aiden. 2017. "De Novo Assembly of the Aedes Aegypti Genome Using Hi-C Yields ChromosomeLength Scaffolds." Science 356: 92-95.

Durand, N.C., M.S. Shamim, I. Machol, S.S.P. Rao, M.H. Huntley, E.S. Lander, and E.L. Aiden. 2016. "Juicer Provides a One-Click System for Analyzing Loop-Resolution Hi-C Experiments." Cell Syst 3: 95-98.

Feuk, Lars, Jeffrey R. MacDonald, Terence Tang, Andrew R. Carson, Martin Li, Girish Rao, Razi Khaja, and Stephen W. Scherer. 2005. "Discovery of Human Inversion Polymorphisms by Comparative Analysis of Human and Chimpanzee DNA Sequence Assemblies." PLoS Genetics 1 (4): e56. https://doi.org/10.1371/journal.pgen.0010056.

Flynn, Jullien M., Robert Hubley, Clément Goubert, Jeb Rosen, Andrew G. Clark, Cédric Feschotte, and Arian F. Smit. 2020. "RepeatModeler2 for Automated Genomic Discovery of Transposable Element Families." Proceedings of the National Academy of Sciences 117 (17): 9451-57. https://doi.org/10.1073/pnas.1921046117.

Fonseca NA, Vieira CP, Schl "otterer C, Vieira J. 2012. The DAIBAM MITE element is involved in the origin of one fixed and two polymorphic Drosophila virilis phylad inversions. Fly 6(2):71-74.

Goidts, Violaine, Justyna M. Szamalek, Pieter J. de Jong, David N. Cooper, Nadia Chuzhanova, Horst Hameister, and Hildegard Kehrer-Sawatzki. 2005. "Independent Intrachromosomal Recombination Events Underlie the Pericentric Inversions of Chimpanzee and Gorilla Chromosomes Homologous to Human Chromosome 16." Genome Research 15 (9): 1232-42. https://doi.org/10.1101/gr.3732505.

González, Josefa, Ferran Casals, and Alfredo Ruiz. 2007. "Testing Chromosomal Phylogenies and Inversion Breakpoint Reuse in Drosophila." Genetics 175 (1): 167-77. https://doi.org/10.1534/genetics.106.062612.

Guillén, Yolanda, and Alfredo Ruiz. 2012. "Gene Alterations at Drosophila Inversion Breakpoints Provide Prima Facie Evidence for Natural Selection as an Explanation for Rapid Chromosomal Evolution." BMC Genomics 13 (1): 53. https://doi.org/10.1186/1471-2164-13-53.

Hoffmann, Ary A., and Loren H. Rieseberg. 2008. "Revisiting the Impact of Inversions in Evolution: From Population Genetic Markers to Drivers of Adaptive Shifts and Speciation?" Annual Review of Ecology, Evolution, and Systematics 39 (December): 21-42. https://doi.org/10.1146/annurev.ecolsys.39.110707.173532.

Hoffmann, Ary A., Carla M. Sgrò, and Andrew R. Weeks. 2004. "Chromosomal Inversion Polymorphisms and Adaptation." Trends in Ecology \& Evolution 19 (9): 482-88. https://doi.org/10.1016/j.tree.2004.06.013.

Keightley, P.D., U. Trivedi, M. Thomson, F. Oliver, S. Kumar, and M.L. Blaxter. 2009. "Analysis of the Genome Sequences of Three Drosophila Melanogaster Spontaneous Mutation Accumulation Lines." Genome Res 19: 1195-1201.

Kim, D., B. Langmead, and S.L. Salzberg. 2015. "HISAT: A Fast Spliced Aligner with Low Memory Requirements." Nat. Methods 12: 357-360.

Kitagawa O, Wakahama KI, Fuyama Y, Shimada Y, Takanashi E, Hatsumi M, Uwabo M, Mita Y. 1982. Genetic study of Drosophila nasuta subgroup, with notes on distribution and morphology. Jpn. J. Genet. 57:113-141.

Kolmogorov, Mikhail, Jeffrey Yuan, Yu Lin, and Pavel A. Pevzner. 2019. “Assembly of Long, Error-Prone Reads Using Repeat Graphs." Nature Biotechnology 37 (5): 540-46. https://doi.org/10.1038/s41587-019-0072-8.

Koren, Sergey, Brian P. Walenz, Konstantin Berlin, Jason R. Miller, Nicholas H. Bergman, and Adam M. Phillippy. 2017. "Canu: Scalable and Accurate Long-Read Assembly via Adaptive k-Mer Weighting and Repeat Separation." Genome Research 27 (5): 722-36. https://doi.org/10.1101/gr.215087.116.

Krimbas, Kōstas V., and Jeffrey R. Powell, eds. 1992. Drosophila Inversion Polymorphism. Boca Raton, Fla: CRC Press.

Kriventseva, Evgenia V, Dmitry Kuznetsov, Fredrik Tegenfeldt, Mosè Manni, Renata Dias, Felipe A Simão, and Evgeny M Zdobnov. 2019. "OrthoDB V10: Sampling the Diversity of Animal, Plant, Fungal, Protist, Bacterial and Viral Genomes for Evolutionary and Functional Annotations of Orthologs." Nucleic Acids Research 47 (D1): D807-11. https://doi.org/10.1093/nar/gky1053.

Krivshenko, J. D. 1963. "The Chromosomal Polymorphism of Drosophila Busckii in Natural Populations." Genetics 48 (9): 1239-58.

Kurtz, S., A. Phillippy, A.L. Delcher, M. Smoot, M. Shumway, C. Antonescu, and S.L. Salzberg. 2004. "Versatile and Open Software for Comparing Large Genomes." Genome Biol 5:R12.

Lambert, D. M. 1982. "Mate Recognition in Members of the Drosophila Nasuta Complex." Animal Behaviour 30 (2): 438-43. https://doi.org/10.1016/S0003-3472(82)80054-7. 
Lemeunier, F., and M. Ashburner. 1984. "Relationships within the Melanogaster Species Subgroup of the Genus Drosophila (Sophophora)." Chromosoma 89 (5): 343-51. https://doi.org/10.1007/BF00331251.

$\mathrm{Li}, \mathrm{H}$. 2016. "Minimap and miniasm: fast mapping and de novo assembly for noisy long sequences." Bioinformatics 32: 2103-2110.

Mai, Dat, Matthew J Nalley, and Doris Bachtrog. 2020. "Patterns of Genomic Differentiation in the Drosophila Nasuta Species Complex." Edited by Stephen Wright. Molecular Biology and Evolution 37 (1): 208-20. https://doi.org/10.1093/molbev/msz215.

McBroome, Jakob, Liang, David and Corbett-Detig Russell, 2020. Fine-Scale Position Effects Shape the Distribution of Inversion Breakpoints in Drosophila melanogaster, Genome Biology and Evolution, Volume 12, Issue 8, Pages 1378-1391, https://doi.org/10.1093/gbe/evaa103

Muller, H. J. 1940. "Bearing of the Drosophila Work on Systematics." The New Systematics, 185-268.

Murphy, William J., Denis M. Larkin, Annelie Everts-van der Wind, Guillaume Bourque, Glenn Tesler, Loretta Auvil, Jonathan E. Beever, et al. 2005. "Dynamics of Mammalian Chromosome Evolution Inferred from Multispecies Comparative Maps." Science (New York, N.Y.) 309 (5734): 613-17. https://doi.org/10.1126/science.1111387.

Papaceit, Montserrat, Montserrat Aguadé, and Carmen Segarra. 2006. "Chromosomal Evolution of Elements B and $\mathrm{C}$ in the Sophophora Subgenus of Drosophila: Evolutionary Rate and Polymorphism." Evolution; International Journal of Organic Evolution 60 (4): 768-81.

Pertea, M., G.M. Pertea, C.M. Antonescu, T.-C. Chang, J.T. Mendell, and S.L. Salzberg. 2015. "StringTie Enables Improved Reconstruction of a Transcriptome from RNA-Seq Reads." Nat. Biotechnol 33: 290-295.

Pevzner, Pavel, and Glenn Tesler. 2003. "Human and Mouse Genomic Sequences Reveal Extensive Breakpoint Reuse in Mammalian Evolution." Proceedings of the National Academy of Sciences 100 (13): 7672-77. https://doi.org/10.1073/pnas.1330369100.

Pope, A. 1987. "Inversion Polymorphism in Species of the Drosophila Nasuta Subgroup from Thailand." Genetica 72 (1): 55-64. https://doi.org/10.1007/BF00126978.

Porubsky, David, Ashley D. Sanders, Wolfram Höps, PingHsun Hsieh, Arvis Sulovari, Ruiyang Li, Ludovica Mercuri, et al. 2020. "Recurrent Inversion Toggling and Great Ape Genome Evolution." Nature Genetics 52 (8): 849-58. https://doi.org/10.1038/s41588-020-0646-x.

Powell, Jeffrey R. 1997. Progress and Prospects in Evolutionary Biology: The Drosophila Model. New York: Oxford University Press.

Ranz, José M., Damien Maurin, Yuk S. Chan, Marcin von Grotthuss, LaDeana W. Hillier, John Roote, Michael Ashburner, and Casey M. Bergman. 2007. "Principles of Genome Evolution in the Drosophila Melanogaster Species Group." PLOS Biology 5 (6): e152. https://doi.org/10.1371/journal.pbio.0050152.

Reis, Micael, Cristina P Vieira, Rodrigo Lata, Nico Posnien, and Jorge Vieira. 2018. “Origin and Consequences of Chromosomal Inversions in the Virilis Group of Drosophila." Genome Biology and Evolution 10 (12): 315266. https://doi.org/10.1093/gbe/evy239.

Richards, Stephen, Yue Liu, Brian R. Bettencourt, Pavel Hradecky, Stan Letovsky, Rasmus Nielsen, Kevin Thornton, et al. 2005. "Comparative Genome Sequencing of Drosophila Pseudoobscura: Chromosomal, Gene, and CisElement Evolution." Genome Research 15 (1): 1-18. https://doi.org/10.1101/gr.3059305.

Ruan, Jue, and Heng Li. 2020. "Fast and Accurate Long-Read Assembly with Wtdbg2." Nature Methods 17 (2): $155-$ 58. https://doi.org/10.1038/s41592-019-0669-3.

Simão, F.A., R.M. Waterhouse, P. loannidis, E.V. Kriventseva, and E.M. Zdobnov. 2015. "BUSCO: Assessing Genome Assembly and Annotation Completeness with Single-Copy Orthologs." Bioinformatics 31: 3210-3212.

Sonoda E, Hochegger H, Saberi A, Taniguchi Y, Takeda S. 2006. Differential usage of non-homologous end-joining and homologous recombination in double strand break repair. DNA Repair 5(9-10):1021-1029.

Sperlich, D., and P. Pfriem. 1986. "Chromosomal Polymorphism in Natural and Experimental Populations." The Genetics and Biology of Drosophila 3: 257-309.

Spieth HT. 1969. Courtship and Mating Behavior of the Drosophila nasuta Subgroup of Species. Univ. Texas Publ. 6918:255-270.

Sturtevant, A. H. 1917. "Genetic Factors Affecting the Strength of Linkage in Drosophila." Proceedings of the National Academy of Sciences 3 (9): 555-58. https://doi.org/10.1073/pnas.3.9.555.

Tesler, Glenn. 2002. "GRIMM: Genome Rearrangements Web Server." Bioinformatics (Oxford, England) 18 (3): 492-93. https://doi.org/10.1093/bioinformatics/18.3.492. 
Vaser, R., I. Sović, N. Nagarajan, and M. Šikić. 2017. "Fast and Accurate de Novo Genome Assembly from Long Uncorrected Reads." Genome Res 27: 737-746.

Vieira, J., C. P. Vieira, D. L. Hartl, and E. R. Lozovskaya. 1997. “Discordant Rates of Chromosome Evolution in the Drosophila Virilis Species Group." Genetics 147 (1): 223-30.

Walker, B.J., T. Abeel, T. Shea, M. Priest, A. Abouelliel, S. Sakthikumar, C.A. Cuomo, Q. Zeng, J. Wortman, and S.K. Young. 2014. "Pilon: An Integrated Tool for Comprehensive Microbial Variant Detection and Genome Assembly Improvement."

Zhang, Z., J. Li, X.-Q. Zhao, J. Wang, G.K.-S. Wong, and J. Yu. 2006. "KaKs_Calculator: Calculating Ka and Ks through Model Selection and Model Averaging." Genomics Proteomics Bioinformatics 4: 259-263.

Zhao, Shaying, Jyoti Shetty, Lihua Hou, Arthur Delcher, Baoli Zhu, Kazutoyo Osoegawa, Pieter de Jong, William C. Nierman, Robert L. Strausberg, and Claire M. Fraser. 2004. "Human, Mouse, and Rat Genome Large-Scale Rearrangements: Stability versus Speciation." Genome Research 14 (10A): 1851-60. https://doi.org/10.1101/gr.2663304.

Zhou, Qi, and Doris Bachtrog. 2012. "Sex-Specific Adaptation Drives Early Sex Chromosome Evolution in Drosophila." Science 337 (6092): 341-45. https://doi.org/10.1126/science.1225385.

Table 1. Inversion sizes along the nasuta phylogeny

\begin{tabular}{cccc}
\hline Inversion & Size & $\begin{array}{c}\text { Genome } \\
\text { Proportion }\end{array}$ & $\begin{array}{c}\text { Chromosome } \\
\text { Proportion }\end{array}$ \\
\hline A1 & $8,435,043$ & 0.050 & 0.251 \\
A2 & $3,942,469$ & 0.024 & 0.117 \\
A3 & $5,566,094$ & 0.033 & 0.166 \\
A4 & $9,812,392$ & 0.059 & 0.292 \\
A5 & $2,884,156$ & 0.017 & 0.086 \\
A6 & $7,149,585$ & 0.043 & 0.213 \\
A7 & $10,244,303$ & 0.061 & 0.305 \\
A8 & $11,286,451$ & 0.067 & 0.336 \\
B1 & $17,991,444$ & 0.107 & 0.590 \\
B2 & $11,126,246$ & 0.066 & 0.365 \\
B3 & $18,041,703$ & 0.108 & 0.592 \\
CD1 & $17,855,989$ & 0.107 & 0.322 \\
CD2 & $15,570,471$ & 0.093 & 0.281 \\
CD3 & $14,885,804$ & 0.089 & 0.268 \\
CD4 & $6,012,219$ & 0.036 & 0.108 \\
CD5 & $7,631,642$ & 0.046 & 0.138 \\
CD6 & $13,246,961$ & 0.079 & 0.239 \\
CD7 & $1,720,990$ & 0.010 & 0.031 \\
CD8 & $10,943,708$ & 0.065 & 0.197 \\
E1 & $10,243,449$ & 0.061 & 0.290 \\
E2 & $10,255,425$ & 0.061 & 0.291 \\
E3 & $5,293,609$ & 0.032 & 0.150 \\
\hline & & & \\
\hline
\end{tabular}


Table S1. Strains investigated

\begin{tabular}{lll}
\hline species & strain & collection location \\
\hline D. albomicans & $15112-1751.00$ & Okinawa, Japan \\
D. nasuta & $15112-1781.00$ & Mysore, India \\
D. kepulauana & $15112-1761.03$ & Ulu Temburong, Borneo \\
D. s. albostrigata & $15112-1771.04$ & Rizal, Phillipines \\
D. s. bilimbata & $15112-1821.10$ & Guam \\
D. s. sulfurigaster & $15112-1831.01$ & Kavieng, New Ireland \\
D. pallidifrons & PN175_E-19901 & Ponape Micronesia \\
\hline
\end{tabular}

Table S2. Inferred approximate inversion breakpoints

\begin{tabular}{lrrr}
\hline & \multicolumn{1}{l}{ Start } & \multicolumn{1}{l}{ End } & \\
Inversion & coordinates & coordinates & Size $(\mathrm{kb})$ \\
\hline Muller_A & 0 & 33597023 & 33,597 \\
A1 & 8461279.5 & 16896322.5 & 8,435 \\
A2 & 27993544 & 31936013 & 3,942 \\
A3* & 25697206.5 & 31263300 & 4,758 \\
A4 & 7083931 & 16896322.5 & 9,812 \\
A5* & 5577123.5 & 8461279.5 & 10,772 \\
A6 & 25697206.5 & 32846791.5 & 7,150 \\
A7 & 22802680.5 & 33046983 & 10,244 \\
A8 & 19976849 & 31263300 & 11,286 \\
Muller_B & 0 & 30469903 & 30,470 \\
B1 & 5356061 & 23347504.5 & 17,991 \\
B2 & 12054629 & 23180875 & 11,126 \\
B3 & 12053716 & 23181816 & 11,128 \\
Muller_DC & 0 & 55495487 & 55,495 \\
CD1 & 13906973 & 31762961.5 & 17,856 \\
CD2 & 6445133 & 22015604 & 15,570 \\
CD3 & 0 & 14885804 & 14,886 \\
CD4 & 42929924 & 48942143 & 6,012 \\
CD5 & 0 & 7631642 & 7,632 \\
CD6 & 19474208 & 32721168.5 & 13,247 \\
CD7* & 31000073 & 32721063 & 5,278 \\
CD8* & 24751727 & 35695434.5 & 14,491 \\
Muller_E & 0 & 35291776 & 35,292 \\
E1 & 16662651 & 26906100 & 10,243 \\
E2 & 9937162.5 & 20192587.5 & 10,255 \\
E3 & 3859696.5 & 9148309 & 5,289 \\
\hline n & & &
\end{tabular}

* nested inversion 